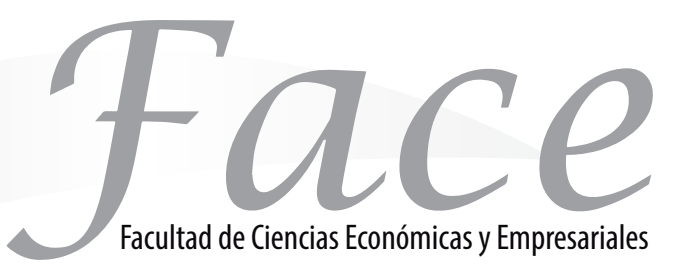

ISSN 1794-9920

Volumen $15 \mathrm{~N}^{\circ} 1$ Año 2015

Págs. 29- 44

\title{
PROCESO DE TOMA DE DECISIONES EN LAS ORGANIZACIONES: UNA MIRADA A LAS INDUSTRIAS DE ALTA TECNOLOGÍA
}

\author{
Alejandro Aristizábal Mesa* \\ Alexandra Montoya . $^{* *}$ \\ Iván Alonso Montoya R. ${ }^{* * *}$
}

Fecha de Recepción: 27 de Abril 2015

Fecha de Aprobación: 15 de Junio 2015

\begin{abstract}
Resumen
El presente artículo señala los aspectos más relevantes alrededor de la toma de decisiones en las organizaciones. Describe la capacidad de absorción, la teoría de la organización y el campo del direccionamiento estratégico como fundamentales en la comprensión del proceso de toma de decisiones en las organizaciones. Adicionalmente, aborda las particularidades que tiene la toma de decisiones en las industrias de alta tecnología, destacando no sólo sus características sino también el proceso llevado a cabo para tener en cuenta no sólo las estrategias emergentes sino también las deliberadas, la metodología seguida es analítica - descriptiva.

Palabras Clave: Toma de decisiones, Innovación, Organización, Alta Tecnología

* Magíster en Ingeniería Administrativa, Ingeniero Químico,Facultad de Minas. cel. 3201614520 E-mail: aaristim@unalmed.edu.co

** PhD, Profesora Asociada, Coordinadora Nacional del Concurso docente, Vicerrectoría General, Carrera 45 No 26-85 - Edificio Uriel Gutiérrez, Bogotá D.C. - Colombia, Tel: 571-3165000 ext. 18364, E-mail: lamontoyar@unal.edu.co, Grupo de investigación Modelamiento y análisis energía ambiente economía.

*** PhD, Profesor Asociado, Facultad de Minas, Doctor en Ciencias Económicas, Magister en Administración, Administrador de empresas. Universidad Nacional de Colombia Carrera 80 No 65-223 - Núcleo Robledo- Medellín - Colombia ext. 45203, cel. 3005632034 E-mail: iamontoyar@unal.edu.co Grupo de investigación Modelamiento y análisis energía ambiente economía.
\end{abstract}




\title{
DECISION-MAKING PROCESS IN THE ORGANIZATIONS: A LOOK AT HIGH-TECH INDUSTRIES
}

\begin{abstract}
Abstrat
This document identifies the main aspects that affect the decision-making process in the organizations. It describes the importance of understanding the absorptive capacity, organizational theory and strategic direction in the organization's decision-making process and addresses it's particularities in the high tech industries, emphasizing its characteristics and the process carried out to take into account, not only the emerging strategies, but also the deliberate ones. The study adopted an analytical and descriptive method.
\end{abstract}

keywords: Decision-making, Innovation, Organization, High-Technology

\section{PROCESSO DE TOMADA DE DECISÃO NAS ORGANIZAÇÕES: UM OLHAR PARA AS INDÚSTRIAS DE ALTA-TECNOLOGIA}

\section{Reama}

Este artigo destaca os pontos maisrelevantes em torno da tomada de decisão nas organizações.Descreve a capacidade de absorção, teoria da organização e o direcionamento estratégico como fundamentais para a compreensão do processo de tomada de decisão nas organizações. Além disso, aborda as particularidades que tem a tomada de decisão em indústrias de alta-tecnologia, destacando não só suas características, mas também o processo realizado para ter em conta as estratégias emergentes e deliberada.O estudo adotou um método analítico e descritivo.

Palavras-Chave: Tomada de Decisões, Inovação, Organização, Alta- Tecnologia.

Claves JEL:

M10 - General

M15 - IT Management

M51 - FirmEmploymentDecisions; Promotions 


\section{Introducción:}

Las empresas pueden ser vistas como sistemas en los cuales, se genera y procesa información, se toman decisiones, se evalúan resultados, y se cambian procedimientos (Cyert \& March, 1992). Esta toma de decisiones es uno de los componentes claves en la teoría de la firma y es el foco de atención principal del direccionamiento estratégico (Montoya \& Montoya, 2003). Reconocer este proceso de toma de decisiones, es fundamental en el desempeño de las organizaciones. En este sentido, uno de los objetivos de la administración estratégica será desarrollar la capacidad de entender por qué algunas empresas tienen éxito mientras que otras fracasan aun cuando tienen numerosos elementos en común (Hill \& Jones, 2005).

Cyert\&March (1992) recopilaron los elementos más relevantes de la teoría de la firma, sugiriendo además propuestas a los diferentes debates que se han generado a lo largo de la construcción de la misma. Dicha teoría contempla la toma decisiones bajo diferentes contextos como se señalará más adelante, mostrando además elementos claves y diferenciadores de la teoría de la organización. Por su parte, Montoya \& Montoya (2003) recopilaron los principales avances alcanzados en el campo del direccionamiento estratégico en relación con la toma de decisiones en las organizaciones. El conocimiento alrededor de una problemática en una organización puede favorecer la toma de decisiones alrededor de la inversión en Investigación y Desarrollo $(I+D)$ y en el aprovechamiento de nuevas tecnologías. Es aquí donde la "capacidad de absorción" juega un papel fundamental en la organización, ya que le permitirá identificar,absorber, asimilar, transformar y aplicar o explotar comercialmente conocimiento obtenido de fuentes externas a la organización (Cohen y Levinthal, 1990; Camisón \&Forés 2014, González et al, 2015. Adicionalmente aquellas empresas pertenecientes a una industria de alta tecnología, se verán obligadas a desarrollar capacidad de absorción superiores que aquellas que pertenecen a industrias tradicionales, en especial, porque deben tener rápida adaptación y procesos de aprendizaje para poder mantenerse en la punta del conocimiento (Spithoven, Clarysse, \& Knockaert, 2010).
Este documento estudia los aspectos más relevantes en la toma de decisiones de las organizaciones para que puedan ser más competitivas en el entorno de la industria de la alta tecnología. Primero retoma el concepto de capacidad de absorción, con el fin de establecer su importancia en la toma de decisiones. Posteriormente se abordan las teorías de la organización y la firma, para luego dar paso al campo del direccionamiento estratégico, dentro del cual se enmarcan las decisiones de la organización. Finalmente, se muestran las particularidades que tiene la toma de decisiones en las empresas de alta tecnología.

Para alcanzar dicho objetivo se organiza la información mediante un proceso analítico descriptivo que parte de la conceptualización de la capacidad de absorción, y el campo del direccionamiento estratégico como fundamentales en la comprensión del proceso de toma de decisiones en las organizaciones. Este punto de partida permite establecer las premisas fundamentales para la aplicación del proceso de toma de decisiones en una industria de alta tecnología.

\section{Marco Teórico :}

\subsection{Hacia una toma de decisiones desde la estrategia}

Para Cohen \&Levinthal (1990), la capacidad de absorción consiste en la habilidad para reconocer el valor de nueva información, asimilarlo y aplicarlo con fines comerciales; sin embargo, existe discrepancia en la literatura acerca de las dimensiones que cubre la capacidad de absorción(Jiménez, García, \& Molina, 2011) y que presentan Hurtado \&Gonzalez (2015) en la Tabla No. 1. 
Tabla N ${ }^{\circ} 1$

Dimensiones de la Capacidad de Absorción

\begin{tabular}{|c|c|}
\hline AUTORES & DIMENSIONES \\
\hline $\begin{array}{l}\text { Cohen and Levinthal (1989), } \\
\text { Cohen and Levinthal (1990), } \\
\text { Mowery and Oxley (1995) } \\
\text { Cockburn and Henderson (1998), } \\
\text { Szulanski (1996), Chen et al. (2014), } \\
\text { Chang et al. (2014), García Morales } \\
\text { et al. (2014) }\end{array}$ & $\begin{array}{l}\text { Identificación } \\
\text { Asimilación } \\
\text { Explotación }\end{array}$ \\
\hline Heeley (1997) & $\begin{array}{l}\text { Adquisición } \\
\text { Difusión } \\
\text { Capacidadestécnicas }\end{array}$ \\
\hline $\begin{array}{l}\text { Lane and Lubatkin (1998), Dyer } \\
\text { and Singh (1998), Minbaeva et al. } \\
\text { (2003), Lane, Koka and Pathak } \\
\text { (2006), Schildt et al. (2012) }\end{array}$ & $\begin{array}{l}\text { Reconocimiento- } \\
\text { evaluación } \\
\text { Asimilación } \\
\text { Aplicación }\end{array}$ \\
\hline $\begin{array}{l}\text { Zahra and George (2002), Jansen } \\
\text { et al. (2005) Vega-Jurado et al. } \\
\text { (2008) Gluch et al. (2009), Flatten } \\
\text { et al. (2011), Jiménez-Barrionuevo } \\
\text { et al. (2011), Datta (2011), Ritala } \\
\text { and Hurmelinna (2013), } \\
\text { Waranantakul and } \\
\text { Ussahawanitchakit (2012) Maynez- } \\
\text { Guaderrama et al. (2012) Gebauer } \\
\text { et al. (2012) Cepeda-Carrión et al. } \\
\text { (2012), Su et al. (2013), Patterson y } \\
\text { Ambrosini (2015), Lao y Lu (2015), } \\
\text { Seo et al. (2015) }\end{array}$ & $\begin{array}{l}\text { Asimilación } \\
\text { Transformación } \\
\text { Explotación } \\
\text { Adquisición }\end{array}$ \\
\hline Todorova and Durisin (2007) & $\begin{array}{l}\text { Reconocimiento } \\
\text { Adquisición } \\
\text { Asimilación } \\
\text { Transformación } \\
\text { Explotación }\end{array}$ \\
\hline Murovec and Prodan (2009) & $\begin{array}{l}\text { Capacidad de } \\
\text { absorcióncientífica } \\
\text { Capacidad de absorción de } \\
\text { Mercado }\end{array}$ \\
\hline Liao et al. (2007) & Evaluación de uso \\
\hline Xiong and Bharadwaj (2011) & $\begin{array}{l}\text { Conocimiento de } \\
\text { absorción de la capacidad } \\
\text { en I + D Conocimiento de } \\
\text { absorción de la capacidad } \\
\text { en la comercialización y del } \\
\text { Cliente }\end{array}$ \\
\hline Hadi et al. (2011) & $\begin{array}{l}\text { La complementariedad de } \\
\text { conocimientos } \\
\text { relacionados experiencia } \\
\text { previa. }\end{array}$ \\
\hline Nugraha (2011) & $\begin{array}{l}\text { Potenciales: los } \\
\text { mecanismos de } \\
\text { organización, las prácticas } \\
\text { de recursos humanos, el } \\
\text { conocimiento atributos } \\
\text { Real: el rendimiento de los } \\
\text { empleados }\end{array}$ \\
\hline
\end{tabular}

Fuente: Hurtado, A., \&Gonzalez-Campo, C. H. (2015). Pág. 20
En general puede decirse que la definición de Jiménez et al, 2011, soluciona estos conflictos al unificarla en la siguiente apreciación:

"Se refiere a la relativa capacidad de la organización para desarrollar una serie de rutinas organizativas y procesos estratégicos a través del cual se adquiere, asimila, transforma y conocimientos adquiridos hazañas de fuera de la organización para crear valor" (Jiménez et al, 2011, Pág.192).

Para Cohen \&Levinthal (1990), la capacidad de absorción de la organización es una consecuencia de la capacidad de absorción individual y la manera como se comunica dicha información dentro de la organización, el conocimiento previo es la base fundamental para la capacidad de absorción de nuevo conocimiento. La experiencia y buen desempeño en una tarea de aprendizaje mejora el desempeño en las tareas siguientes, se "aprende a aprender", puesto que se tienen que aprender menos aspectos nuevos para adquirir otros conocimientos. De igual manera, la solución de problemas sigue el mismo comportamiento. En ninguno de los casos basta con exponer al individuo durante poco tiempo al conocimiento previo, es necesario que éste sea realmente aprendido de manera que constituya una base sólida para la entrada del nuevo conocimiento.

De esta manera, es posible afirmar que el aprendizaje es acumulativo y el desempeño será mejor cuando está íntimamente relacionado con el conocimiento existente. La diversidad de conocimiento, por su parte, facilita el proceso de innovación por medio de asociaciones y vínculos por parte del individuo (Cohen \& Levinthal, 1990).

De acuerdo con este análisis, podría llegarse a pensar que la capacidad de absorción de la organización es una función de las capacidades individuales de sus miembros. Sin embargo, la capacidad de absorción organizacional no es la mera suma de las capacidades individuales, puesto que es necesario que la organización esté en capacidad de utilizar con fines comerciales el conocimiento adquirido por sus individuos. Lo clave en este punto es identificar la existencia de dos tipos de interfaces para el intercambio de conocimiento en la 
organización: la interface externa en la cual se da adquisición de conocimiento a partir del medio, y las interfaces internas en las cuales se intercambia conocimiento entre los individuos que conforman la organización (Cohen \& Levinthal, 1990)

Las estructuras de comunicación resultan claves para mejorar la manera como el intercambio de conocimiento se da en cada una de las interfaces mencionadas. Cohen \&Levinthal (1990) sugieren lo siguiente:

- Si el conocimiento interno de la organización difiere mucho del conocimiento del medio, lo mejor es nombrar ciertos "guardianes" que se encargarán de adquirir el conocimiento externo y divulgarlo al interior de la organización (Medina,\&Verástegui, (2015).

- Si por el contrario, el conocimiento externo está ítnimamente relacionado con las actividades en ejecución, no es necesario tener guardianes puesto que la transferencia se dará sin dificultades.

- Si la organización se encuentra en una industria con un entrorno de cambios rápidos y no se sabe de dónde pueda provenir nuevo conocimiento, los guardianes no resultan adecuados. Es necesario definir un grupo más amplio de receptores de nuevo conocimento.

En cualquiera de los casos, el punto principal es el nivel de conocimiento que posean los miembros de la organización. Si los guardianes tienen mucho más conocimiento que los otros miembros, la comunicación no será efectiva y por lo tanto la capacidad de absorción organizacional tampoco lo será.

Una buena eficiencia de comunicación sugiere ciertos solapamientos de conocimiento ( Cruz et al, 2007) entre los miembros, puesto que sin estos solapamientos la comunicación no será efectiva. Una distribución Taylorista (Taylor, 1981) del trabajo, generará una diferencia muy grande entre el tipo de conocimiento de los individuos y por lo tanto, fallas en la comunicación. Por su parte, la diversidad en el conocimiento es fundamental en los procesos de innovación. Como ejemplo de ello, puede tomarse un individuo A que forma parte del área de I+D y el individuo $\mathrm{B}$ que forma parte del área comercial,; resulta claro cómo ambos individuos deben tener tanto conocimiento común en diferentes aspectos, pero a su vez diversidad que permita que cada uno haga aportes significativos en las actividades de innovación de la organización.

Como se mencionó anteriormente, las bases de conocimiento previo son fundamentales para adquirir nuevo conocimiento. Dos ideas fundamentales se desprenden de lo anterior (Cohen \& Levinthal, 1990):

- La acumulación de capacidad de absorción durante un período permite una mayor eficiencia de acumulación y,

- La experticia adquirida permite a la firma evaluar el surgimiento de nuevas tecnologías en campos relacionados. Lo cual resulta clave en ambientes inciertos, en los cuales es necesario predecir los avances tecnológicos con la mayor certidumbre posible (González\& Ayala, 2014) .

De esta manera, la acumulabilidad y el efecto en la formación de expectativas, hacen que la capacidad de absorción sea dependiente de la trayectoria o historia organizacional (Cohen \& Levinthal, 1990). Esto tiene una implicación directa en la toma de decisiones gerenciales, puesto que la falta de inversión en capacidad de absorción en un determinado período, hará más costosa y menos atractiva la inversión para el período siguiente, aun teniendo conciencia de cambios tecnológicos significativos en el entorno. Las empresas con buena capacidad de absorción resultan siendo entonces proactivas, mientras que las que tienen baja capacidad de absorción son reactivas ante los cambios tecnológicos.

Cohen \&Levinthal (1990) demostraron cómo se relacionan diversos aspectos de la capacidad de absorción con la I+D. La "oportunidad tecnológica" (Olalla et al, 2014) representa el costo para la empresa de adquirir avance tecnológico. Depende de la cantidad de conocimiento externo disponible y del grado al cual el nuevo conocimiento mejora el desempeño financiero de la empresa. Los incentivos para el aprendizaje (o para crear capacidad de absorción) por medio de inversión en I+D (González, 2014), el cual depende de la cantidad de conocimiento para ser asimilado y explotado 
(mientras más, mayor incentivo), y de la dificultad de aprender (mientras más difícil, mayor inversión en I+D será necesaria). En ambientes en que la información sea muy difícil de asimilar, la I+D tiene un efecto marginal alto en la acumulación de capacidad de absorción(Cohen \& Levinthal, 1990).

Un aspecto clave de la I+D en la acumulación de capacidad de absorción es que contraresta la dificultad que implican los spillovers, (Oliver, Ripoll, 2014) puesto que se puede apropiar de las nuevas tecnologías con mayor facilidad. En muchos casos el conocimiento en ciencias básicas como matemáticas, física, química, etc., es sumamente necesario para poder lograr una mayor apropiación de dichos spillovers(Cohen \& Levinthal, 1990).

1. Decisiones en las empresas y organizaciones: acercamiento a las teorías de la firma y la organización

La diferencia existente entre el proceso real de toma de decisiones en las organizaciones complejas y las explicaciones teóricas desde el punto de vista económico han sido un tema sumamente controversial. Los desacuerdos están centrados en: (i) cuál es la teoría, (ii) su alcance y (iii) los métodos apropiados para mejorarla (Cyert \& March, 1992).

En términos generales, se acepta como objetivo principal de la firma maximizar el rendimiento financiero de cara a los precios establecidos y una función de producción determinada por la tecnología. La maximización consiste en encontrar la combinación óptima de productos y factores (conocido como el punto de equilibrio)(Jiménez, 2014).

Muchos académicos manifiestan inconformidad con estos modelos matemáticos preestablecidos, porque consideran que las suposiciones en los aspectos motivacionales y cognitivos de la firma no son realistas, y que la "firma" de la teoría tiene pocas características de las firmas reales (Cyert \& March, 1992).

Respecto a la toma de decisiones, la teoría ha sido criticada debido a que no ve a la firma como una organización y que los procesos de toma de decisiones como tal no son realistas: las empresas no igualan los costos marginales con las ganancias marginales para decidir la producción y el precio, en lugar de esto siguen alguna regla conocida para ello (Dos Santos, et al 201, Del Angel, et al, 2015). Sin embargo, Cyert\&March (1992) afirman que muchas de las inconformidades hacia la teoría han resultado de una confusión respecto a lo que pretende la teoría: la manera como se adjudican recursos por un sistema de precios. Los autores en cuestión plantean tres características de la teoría de la firma que deben ser enfatizadas y que pueden observarse en la Figura No. 1.

\section{Figura $\mathrm{N}^{\circ} 1$.}

\section{Características de la Teoría de la Firma}

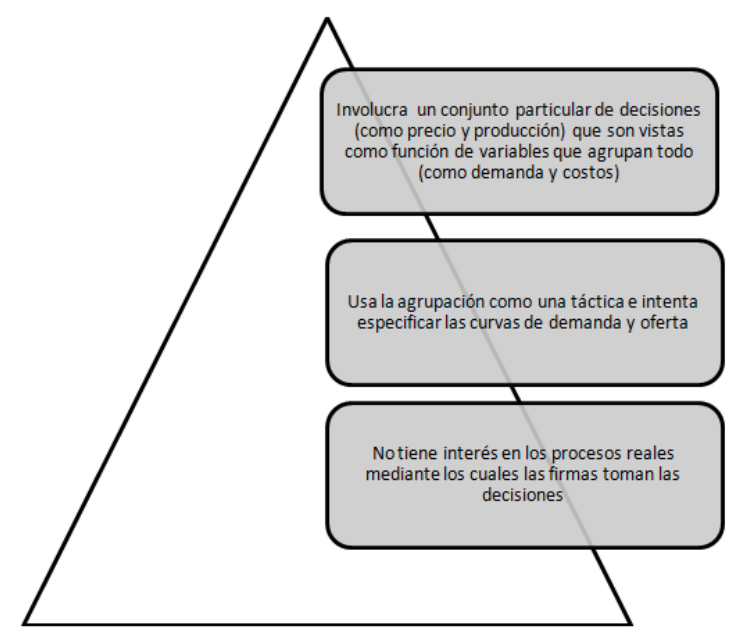

Fuente: Elaboración Propia

Por su parte, el direccionamiento estratégico se estableció como un campo académico en los años sesenta, sin embargo su enseñanza se basaba en el estudio de casos por lo cual se carecía de un fundamento real en el área. Hacia los años setenta comienzan las investigaciones formales y se crean diferentes Journals, generando así conocimientos más formales al respecto (Montoya \& Montoya, 2003) que se pueden observar en la Tabla No. 2 
Figura $\mathrm{N}^{\circ} 2$

Elementos del Proceso estratégico

Se destacan los trabajos de los "pioneros" de la estrategia: Chandler, Ansoff, Andrews y Henderson. Posteriormente se destacan los trabajos de Porter, Parhalad, Barlett, entre otros (Montoya \& Montoya, 2003).

Es así como se deriva el proceso estratégico como la función de tres procesos que centralizan este direccionamiento que se describen en la figura No. 2 y en la tabla No. 3 .

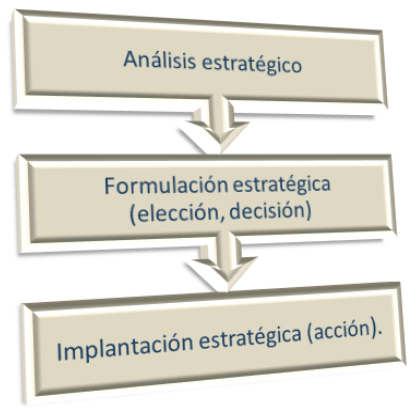

Fuente: Adaptado de Castro, 2010

Tabla $\mathrm{N}^{\circ} 3$

\section{Descripción de las etapas del proceso}

\begin{tabular}{|c|c|}
\hline $\begin{array}{l}\text { Análisis } \\
\text { estratégico }\end{array}$ & $\begin{array}{l}\text { Johnson y Scholes (2001, Pág. 17), "el análisis estratégico consiste en comprender la } \\
\text { posición estratégica de la organización en función de su entorno externo, sus recursos y } \\
\text { competencias internas, y las expectativas e influencias de los Stakeholders". Según Dess y } \\
\text { Lumpkin (2003), el análisis estratégico consiste en el trabajo previo que se debe realizar } \\
\text { para la formulación e implantación eficaz de las estrategias. Para estos autores un análisis } \\
\text { completo debe involucrar tanto factores externos como internos que incluyen los } \\
\text { siguientes procesos: a) analizar los propósitos y los objetivos organizativos: la visión, misión } \\
\text { y objetivos estratégicos de la empresa; b) analizar el entorno: el análisis del entorno permite } \\
\text { obtener información valiosa para la identificación de oportunidades y amenazas; c) realizar } \\
\text { el análisis interno: este proceso ayuda a identificar las fortalezas y debilidades que pueden, } \\
\text { en parte, determinar el éxito de una empresa en un sector; d) valorar los activos intangibles } \\
\text { de la empresa: el capital humano y otros activos intelectuales o intangibles, como son redes } \\
\text { y relaciones entre sus empleados, clientes, proveedores y aliados. }\end{array}$ \\
\hline $\begin{array}{l}\text { Formulación } \\
\text { estratégica } \\
\text { (elección, } \\
\text { decisión) }\end{array}$ & $\begin{array}{l}\text { Para David (1997, Pág. 5), 2008, la formulación de una estrategia consiste en elaborar la } \\
\text { misión de la empresa, detectar las oportunidades y las amenazas externas de la } \\
\text { organización, definir sus fuerzas y debilidades, establecer objetivos a largo plazo, generar } \\
\text { estrategias alternativas y elegir las estrategias concretas que se seguirán". } \\
\text { De acuerdo con Wheelen y Hunger (2007, Pág. 12), la formulación estratégica es el } \\
\text { "desarrollo de planes a largo plazo para administrar de manera eficaz las oportunidades y } \\
\text { amenazas ambientales con base en las fortalezas y debilidades. David (2003), Mintzberg } \\
\text { (1990), Dess y Lumpkin (2003) coinciden en que la formulación de estrategias debe ser un } \\
\text { proceso racional, explícito y sencillo. } \\
\text { Harrison y St. John ( } 2002 \text {, Pág. } 6 \text { ) expresan que "la implantación de la estrategia implica un } \\
\text { patrón de decisiones y acciones destinadas a la consecución de un plan. La implantación de } \\
\text { la estrategia abarca la creación de las estrategias funcionales, los sistemas, las estructuras y } \\
\text { los procesos necesarios para que la organización alcance sus fines estratégicos". Para Dess y } \\
\text { Lumpkin (2003, Pág. 16), la implantación estratégica requiere asegurar que la empresa } \\
\text { posee adecuados controles estratégicos y diseńos organizativos. Según Johnson y Scholes } \\
\text { (1997), la implementación estratégica comprende la planificación de aquellas tareas } \\
\text { relacionadas con la forma de efectuar la elección estratégica y la dirección de los cambios } \\
\text { requeridos; la implementación suele involucrar una planificación detallada de recursos; es } \\
\text { necesario adaptar los sistemas utilizados para dirigir la organización y así lograr conseguir } \\
\text { los aspectos operativos claves }\end{array}$ \\
\hline
\end{tabular}

Fuente: Adaptado de Castro, 2010 
Alejandro Aristizábal Mesa - Alexandra Montoya R. - Iván Alonso Montoya R.

La fijación de metas es el primer paso para la definición de estrategias en las empresas y la consecuente toma de decisiones. Existen dos maneras clásicas de determinar las metas de la organización(Hill \& Jones, 2005). La primera consiste en determinarlas con base en las metas del empresario, la segunda en lograr un consenso compartido por los diversos miembros de la organización. Depende de la estrategia a seguir, en las cuales ambas tratan de definir una orden preferencial de unión para la coalición y en la mayoría de los casos las metas se convierten en una mera aspiración en lugar de un objetivo necesario (Cyert \& March, 1992). Las tres etapas de este proceso se muestra en la Figura 3, primero con un proceso de negociación, luego con un proceso de control y finalmente con un proceso de ajuste.

Figura $\mathrm{N}^{\circ} 3$.

Proceso de Definición de Metas

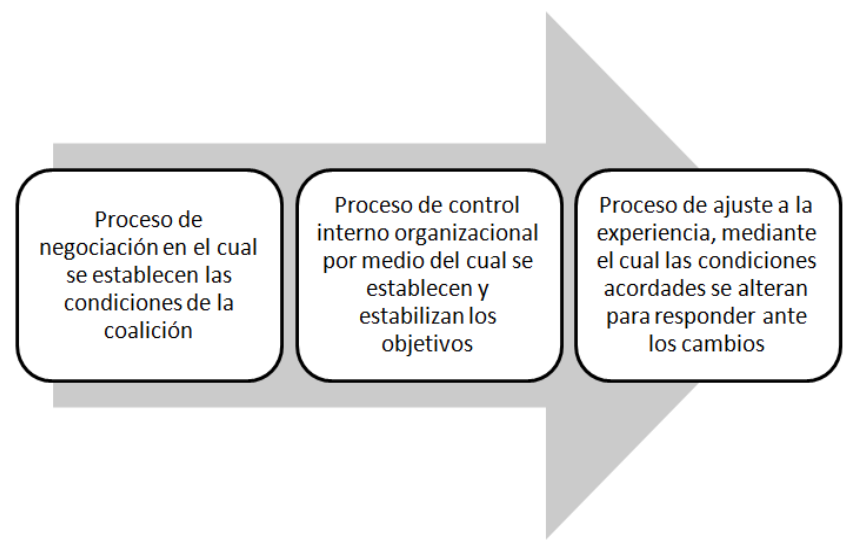

Fuente: Adpatado de Cyert \& March, (1992).

Las metas que se definen en una organización son de diferentes tipos, cada uno de ellos aporta a distintas áreas de la organización, esto posibilita lograr una ventaja competitiva en su industria(Cyert \& March, 1992):

- Meta de producción: para garantizar la estabilidad y cantidad de la producción

- Meta de inventarios: para asegurar la cantidad adecuada de inventarios

- Meta de ventas: en la cual se fija un nivel de ventas deseado

- Meta de participación de mercado: alternativa a la meta de ventas

- Meta de ganancias: o de retorno de la inversión.
Mintzberg(1987) afirma que existen diferentes maneras de aludir a la estrategia explícita, desarrollada desde un propósito de manera intencional, y realizada con anticipación a una decisión específica a la cual aplica, que puede verese en la Figura No. 4 con tres posibilidades como un plan, un patrón o una perspectiva(Montoya, 2009) ( Figura No. 4) :

Figura $\mathrm{N}^{\circ} 4$

Estrategia explícita

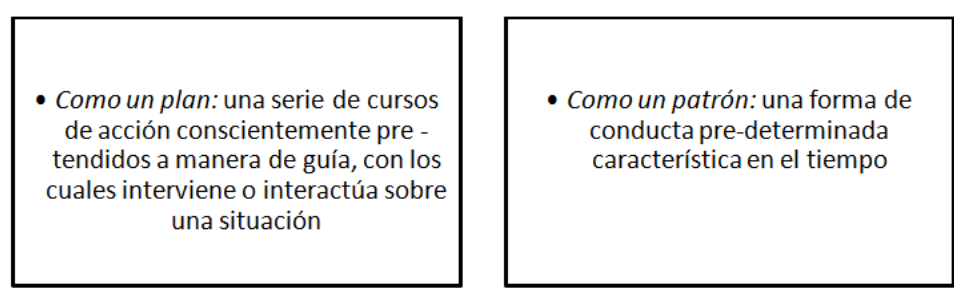

- Como una perspectiva: la cual

orienta y da coherencia a las

actuaciones de la organización en el tiempo

Fuente: Adaptado de Montoya, 2009.

Sin embargo Montoya (2009) sostiene que el punto de vista explícito y planeado para la estrategia no es suficiente, y que es necesario considerar la estrategia como un fenómeno perceptual. La estrategia es entonces para Mintzberg:"un patrón en un flujo de decisiones, con la anotación que una decisión es definida como un compromiso a la acción"( Mintzberg, 1978).

SegúnMintzberg (1978), la estrategia final es la suma de dos sub-estrategias (Montoya, 2009), ver Figura 5:

- Estrategias deliberadas: estrategias intencionadas que resultan realizadas.

- Estrategias emergentes: estrategias realizadas que no fueron intencionadas 
Figura $\mathrm{N}^{\circ} 5$.

Estrategias Deliberadas y Emergentes.

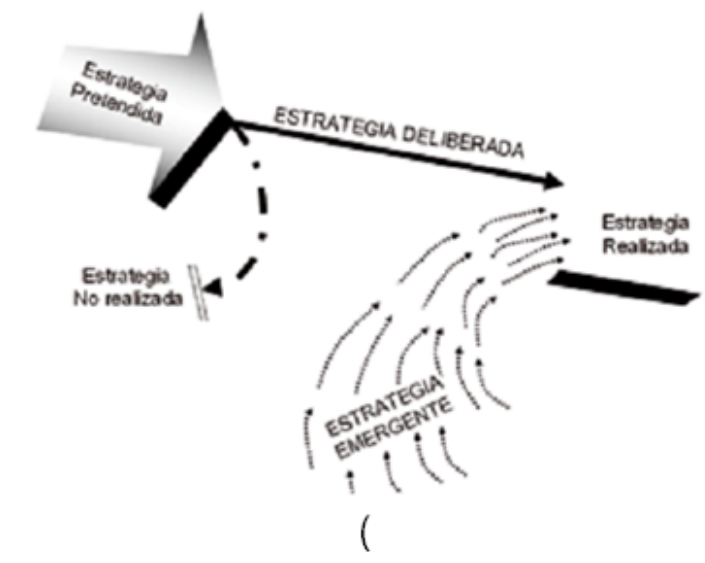

Fuente: Mintzberg (1987, Pág. 14)

Adicionalmente, Mintzberg propone ciclos, es decir, la manera de combinar experiencias con expectativas y como puntos de partida para la formación de estrategias. Tales ciclos son retroalimentaciones de procesos de aprendizaje, de manera que constituyen un proceso de formación estratégica mucho más realista. Mintzberg reconoce los siguientes ciclos (Montoya, 2009):

- Ciclos de convergencia y divergencia

- Ciclos de vida de la estrategia y oleadas de cambio periódico

- Ciclos de configuraciones estructurales

- Ciclos concéntricos y circunferenciales

El impacto de la de estrategia emergente en la toma de decisiones es de suma importancia, puesto que una empresa se puede adaptar a las condiciones del medio a través de la misma.

\section{Y con respecto a las industrias de "alta tecnología"}

Un entorno de "alta tecnología", frecuentemente requiere un estilo de toma de decisiones y estructuras organizacionales diferentes respecto a los entornos de "baja tecnología" (Covin \& Slevin, 2001). Una empresa de "alta tecnología", es la que (Von Glinow, Young, \& Mohrman., 1990; citado en, Doran \& Gunn, 2002):

- Opera en una industria que emplea personal altamente calificados (científicos, tecnólogos e ingenieros)
- Tiene una relación más alta de gastos en $I+D$ respecto a las ventas que en las compañías tradicionales (normalmente 10 veces mayor)

- Se caracteriza por el hecho de que la nueva tecnología hace obsoleta a la existente rápidamente

- Tiene una tasa de crecimiento más alta debido al reforzamiento tecnológico: emergen nuevos productos y se diseñan nuevos procesos.

En las empresas de alta tecnología, el estilo para la toma de decisiones suele ser intuitivo (Covin \& Slevin, 2001; Doran \& Gunn, 2002). Covin \& Slevin (2001), encontraron que para estos casos, es mejor seguir una estructura organizacional orgánica. Sin embargo, si la estructura organizacional es mecanicista, puede resultar adecuado pasar a un estilo tecnocrático para la toma de decisiones por parte del gerente. Jones et al. (1991), demostraron cómo las decisiones centralizadas en las organizaciones pertenecientes a industrias de alta tecnología resultan en una perspectiva sistemática cerrada, problemas políticos internos y bajo desempeño financiero. De acuerdo al estudio realizado por Doran \& Gunn (2002), el empoderamiento de los empleados es clave en las empresas de alta tecnología. Los gerentes no están involucrados en los procesos del día a día; no dicen cómo se deben hacer las cosas, sino qué se debe hacer (Doran \& Gunn, 2002). Para facilitar la toma de estas decisiones, existen modelos que permiten predecir la factibilidad financiera de cada una de ellas, con el fin de disminuir la incertidumbre que caracteriza las industrias de alta tecnología (Marquez \& Blanchar, 2006).

Las empresas de una misma industria, geográficamente cercanas entre sí, tienen un gran acercamiento cognitivo debido a experiencias compartidas (De Jong \& Freel, 2010). Si se presenta un acercamiento excesivo, se limita la innovación puesto que queda poco por aprender. Sin embargo, a medida que la empresa aumenta su capacidad de absorción, también aumenta su acercamiento cognitivo con otros aliados, incluyendo a aquellos más distantes(De Jong \& Freel, 2010).

La capacidad de absorción juega un papel tan importante en las empresas de alta tecnología, que es precisamente uno de los aspectos clave que las 
diferencia de las industrias tradicionales (Spithoven, Clarysse, \& Knockaert, 2010). En las industrias de alta tecnología se tiene entonces la necesidad de generar una capacidad de absorción superior, que permita superar las fronteras geográficas e incentivar las decisiones favorables para los períodos subsecuentes.

Numerosas industrias de alta tecnología tienen la característica de que los costos fijos son altos, asociados principalmente al desarrollo de los productos y publicidad. Por el contrario, los costos de producción de unidades adicionales pueden tender a ser tienden marginales o nulos. De esta manera, la toma de decisiones y las rutinas organizacionales estarán enfocadas en lograr escalas que permitan distribuir los costos fijos en toda la producción y lograr un precio unitario menor que los competidores(Hill \& Jones, 2005).

Las empresas establecidas en una industria de alta tecnologíadeben ajustarse a los cambios tecnológicos de su entorno en caso de aparecer tecnologías disruptivas. Rothaermel \& Thursby (2007), estudiaron el efecto de la nanotecnología y biotecnología sobre firmas establecidas:

"La habilidad de la firma para poder explotar los nuevos métodos de invención dependen inicialmente del acceso a conocimiento tácito acerca de cómo emplear los nuevos métodos, pero con el tiempo, a medida que las firmas aprenden y el conocimiento se codifica (en rutinas o equipos disponibles comercialmente), los resultados de la invención se vuelven más dependientes de inversiones tradicionales en $1+D^{\prime \prime}$ (Rothaermel \& Thursby, 2007,846)

Uno de los retos que enfrentan las industrias de alta tecnología es sobrevivir frente a estas tecnologías disruptivas. Si bien la miopía tecnológica (enfocarse en lo puramente tecnología, olvidando las necesidades del cliente), es una causa de fracaso de la estrategia de muchas empresas de alta tecnología (Hill \& Jones, 2005); el otro extremo es aún más común, es decir, las empresas se limitan a satisfacer las necesidades de los clientes y pasan de alto las tecnologías emergentes, aun siendo conscientes de su existencia(Christensen, 1997). De una manera más amplia, las siguientes son las razones por la cual las empresas establecidas fracasan frecuentemente ante la aparición de una tecnología disruptiva (Pandza \& Holt, 2007):

- Anclaje fuerte a las expectativas de los consumidores

- Condiciones de la demanda

- Inhabilidad para reconfigurar la base de conocimiento debido a la inercia

- Estructuras cognitivas gerenciales incapaces de identificar las nuevas tecnologías

- Una mezcla de factores económicos, organizaciones y estratégicos

En estos casos, la toma de decisiones se deben orientar hacia la inversión en tecnologías potencialmente disruptivas y en tener acceso a los conocimientos relativos a la manera en que las tecnologías disruptivas pueden revolucionar los mercados (Hill \& Jones, 2005). Las herramientas de prospectiva, vigilancia tecnología e inteligencia competitiva resultan siendo claves para poder predecir con éxito el surgimiento de las tecnologías disruptivas.

Por su parte, los nuevos participantes (o atacantes) tienen la ventaja de enfocarse en las oportunidades que ofrece la nueva tecnología disruptiva; ya que no cuentan con presiones para continuar con un modelo desactualizado de negocios, y tampoco tienen que preocuparse por su relación con los clientes y proveedores establecidos. Sin embargo, los nuevos participantes también enfrentan problemas, asociados principalmente a la falta de capital, crecimiento rápido de la organización y necesidad de encontrar la manera de desarrollar la tecnología. En este último punto, las decisiones suelen estar encaminadas a desarrollar la tecnología por sí misma en caso de tener los recursos necesarios, o formar una alianza estratégica con una empresa más grande para poder acceder a los recursos necesarios para llevar a cabo la tarea. La Figura 7 resume estos aspectos, nótese que la identificación de tecnologías disruptivas en una industria puede estar directamente con estrategias emergentes, mientras que si las condiciones en la industria son estables las estrategias planeadas serán las que predominarán. 
Figura $N^{\circ} 6$

\section{Proceso de Toma de Decisiones en las Industrias de} Alta Tecnología

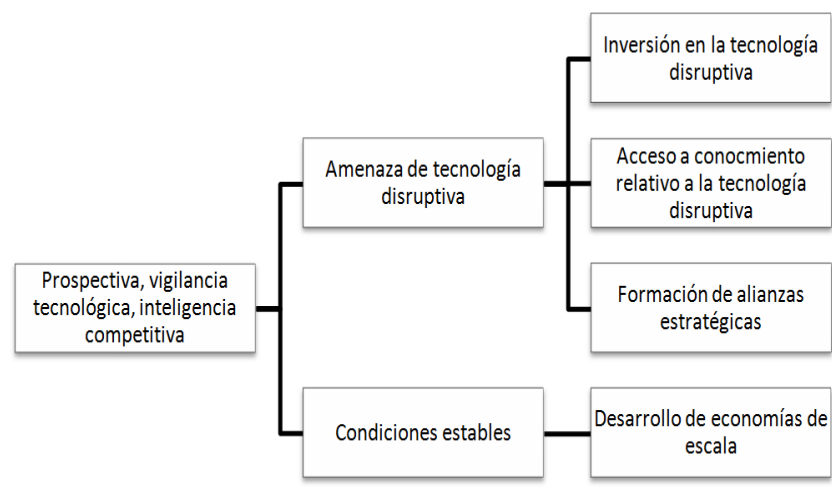

Fuente: Elaboración Propia

\section{Conclusiones:}

La capacidad de absorción de una organización es un determinante clave en la toma de decisiones al interior de la misma, en este sentido, la estrategia realizada por una organización es el resultado de la estrategia deliberada y la estrategia emergente, que responde a los cambios en el entorno. El estilo para la toma de decisiones en las empresas de alta tecnología suele ser intuitivo, con un alto empoderamiento por parte de los empleados. Enfocarse únicamente en los aspectos tecnológicos o, por el contrario, enfocarse exclusivamente en los aspectos de mercado (necesidades actuales de los clientes), son causas de fracaso comunes en las industrias de alta tecnología. Ante la aparición de nuevas tecnologías, la toma de decisiones en las organizaciones establecidas se debe orientar hacia la inversión en tecnologías potencialmente disruptivas y en tener acceso a los conocimientos relativos a la manera en que las tecnologías disruptivas pueden revolucionar los mercados.

\section{Referencias:}

Ansoff, I. H. (1997). La dirección estratégica en la práctica empresarial (2a Ed.). México: Pearson.

Ansoff, I. H., Declerck, R. P. \& Hayes R. L. (1988). El planteamiento estratégico: Nueva tendencia de la administración. México: Trillas.

Camisón Zornoza, C., \&Forés Julián, B. (2014). Capacidad de absorción: antecedentes y resultados.

Castro, A. A. (2010). Direccionamiento estratégico y crecimiento empresarial: algunas reflexiones en torno a su relación. Pensamiento\&Gestión, (28), 85-106.

Cepeda-Carrion, G., Cegarra-Navarro, J., and JimenezJimenez, D. (2012). The Effect of Absorptive Capacity on Innovativeness: Context and Information Systems Capability as Catalysts. British

Certo, S. (2001). Administración moderna: diversidad, calidad, ética y el entorno global. (8a Ed.). Colombia: Pearson Education.

Chang, Ch., Chen, Y., and Lin, J. (2014). The impacts of different $R \& D$ organizational structures on performance of firms: Perspective of absorptive capacity. R\&D Management, 44(5), 466-483.

Chen, H., Qiao, S., and Lee, A. (2014). The impacts of different $R \& D$ organizational structures on performance of firms: Perspective of absorptive capacity. Journal of High Technology Management Research, 25, 83-95.

Christensen, C. M. (1997).The Innovator's Dilemma. Boston, Massachusetts: Harvard Business School Press.

Cockburn, I., Henderson, R. and Stern, S. (2000). Untangling the origins of competitive advantage. Strategic Management Journal, 21, 1123-1145.

Cohen, W. M., \&Levinthal, D. A. (1990). Absorptive Capacity: A New Perspective on Learning and Innovation. Adimistrative Science Quarterly, 35(1), 128-152.

Cohen, W., and Levinthal, D. (1989). Innovation and learning: The two faces of $R \& D$, Economic Journal, 99 (297), 569-596.

Cohen, W., and Levinthal, D. (1990). Absorptive capacity: A new perspective on learning and innovation, Administrative Science Quarterly, 35 (1), pp. 128-152.

Covin, J., \&Slevin, D. (2001). Strategic decision making in an intuitive vs .technocratic mode: structural 
Alejandro Aristizábal Mesa - Alexandra Montoya R. - Iván Alonso Montoya R.

and environmental considerations. Journal of Business Research, 52(1), 51-67. doi:10.1016/S0148-2963(99)00080-6

Cruz, A. M. B., Canino, R. M. B., \& Almeida, D. J. G. (2007). La influencia de la capacidad de absorción en la transferencia de conocimiento interorganizativa. In El comportamiento de la empresa ante entornos dinámicos: XIX Congreso anual y XV Congreso Hispano Francés de $\operatorname{AEDEM}(p$. 52). Asociación Española de Dirección y Economía de la Empresa (AEDEM).

Cyert, R. M., \& March, J. G. (1992). A behavioral theory of the firm (Second Edi.). Malden, Massachussetts: BLackwell. Retrieved from http://scholar.google.com/scholar?hl=en\&btnG $=$ Search\&q=intitle:A+Behavioral+Theory+of+th e+Firm\#0

Datta, A. (2011). Combining Networks, Ambidexterity and Absorptive Capacity to Explain Commercialization of Innovations: A Theoretical Model from Review and Extension. Journal of Management and Strategy, 2 (4); December 2011.

David, F. R. (1997). Concepto de administración estratégica (5a Ed.). México: Prentice Hall Hispanoamericana.

David, F. R. (2008). Conceptos de administración estratégica. (11a Ed.). México: Pearson Education.

De Jong, J. P. J., \&Freel, M. (2010).Absorptive capacity and the reach of collaboration in high technology small firms. Research Policy, 39(1), 47-54. doi:10.1016/j.respol.2009.10.003

Del Angel, L. E. A., Maldonado-Radillo, S. E., \& MáynezGuaderrama, A. I. (2015, January). LA VENTAJA COMPETITIVA, DESDE LA TEORÍA DE RECURSOS Y CAPACIDADES/COMPETITIVE ADVANTAGE FROM RESOURCE THEORY AND CAPABILITIES. In Global Conference on Business \& Finance Proceedings (Vol. 10, No. 1, p. 1278). Institute for Business \& Finance Research.

Dess G. G. \& Lumpkin G. T. (2003).Dirección estratégica: creando ventajas competitivas. Madrid: McGraw Hill.

Dixit, A. K. \&Nalebuff, B. J. (1991).Pensar estratégicamente: un arma decisiva en los negocios, la política y la vida diaria. España: Antoni Bosch.

Doran, G. T., \&Gunn, J. (2002). Decision making in hightech firms: Perspectives of three executives. Business Horizons, 45(6).
Dos Santos, J. G. C., Calíope, T. S., \& Coelho, A. C. (2015). Teorias da Firma como fundamento para formulação de teoriascontábeis. Revista de Educação e PesquisaemContabilidade (REPeC), 9(1).

Dyer J., and Singh, H. (1998). The relational view: cooperative strategies and sources of interorganizational competitive advantage. Academy of Management Review 23(4), 660679.

Flatten, T., Engelen, A., Zahra, A., and Brettel, M. (2011). A measure of absorptive capacity: Scale of development and validation. European Management Journal. 29, 98-116.

Fuentelsaz, L., Polo, Y. \&Maicas, J.P. (2003). Economía digital y estrategia empresarial: un análisis desde la dirección estratégica. Revista de Empresa, 5, 57.

García-Morales, V., Bolívar-Ramos, M., Martín-Rojas, R. (2014). Technological variables and absorptive capacity's influence on performance through corporate entrepreneurship. Journal of Business Research, 67 (7), 1468-1477.

Gluch, P., Mathias, G., and Liane, T: (2009). An absorptive capacity model for green innovation and performance in the construction industry. Construction Management and Economics 27, 451-464.

González, I. (2014). Modelo teórico de capacidad de absorción, innovación organizacional y emprendimiento. Informe de Investigaciones Educativas,27(1), 290-340.

González, R. M., Álvarez, J. A. R., \& Leiva, J. C. (2015). Propuesta de un índice para medir la capacidad de absorción de las MIPYMES costarricenses (Proposalforanindex to measuretheabsorptioncapacity of Costa RicanSMEs). Tec Empresarial, 9(1), 7-18.

González-Campo, C. H., \& Ayala, A. H. (2014). Influencia de la capacidad de absorción sobre la innovación: un análisis empírico en las mipymes colombianas. EstudiosGerenciales, 30(132), 277286.

Goodstein, L. D., Nolan T. M. \& Pfeiffer J. W. (1998).Planeación estratégica aplicada. Bogotá: McGraw-Hill.

Hadi, Z., Farzam, V., Satarifar, M., and Bakhshi, L. (2011).The Relationship between Knowledge Transfer and Competitiveness in "SMES" with Emphasis on Absorptive Capacity and Combinative Capabilities. International Business and Management, 2 (1), 59-85. 
Harrington, H. J. (2005). Administración total del mejoramiento continuo -la nueva generación-. Colombia: McGrawHill

Harrison, J. S. (2002). Fundamentos de la dirección estratégica ( $2 \mathrm{a}$ ed.) Madrid: Internacional Thomson Editores.

Heeley, M. (1997). Appropriating rents from external knowledge: the impact of absorptive capacity on firm sales growth and research productivity. Frontiers of Entrepreneurship Research, 17,390404.

Hill, C. y Jones, G. (2005). Administración estratégica: un enfoque integrado. (6a ed., pp.344-345). México: McGraw-Hill.

Hill, C., \& Jones, G. (2005). Administración Estratégica (6th ed.). México: McGraw-Hill.

Hill, W. L. \& Jones, G. R. (1996).Administración estratégica: un enfoque integrado. (3a Ed.). Bogotá: McGrawHill.

Hurtado, A., \&Gonzalez-Campo, C. H. (2015). MEDICION DE LA CAPACIDAD DE ABSORCION: UN INDICADOR ESTIMADO PARA LOS SECTORES DE MANUFACTURA Y SERVICIOS EN COLOMBIA. Globalización, Competitividad y Gobernabilidad de Georgetown/Universia, 9(2).

Jansen, J., Van Den Bisch, F., and Volberda, H. (2005). Managing potential and realized absorptive capacity: How do organizational antecedents matter? Academy of Management Journal, 48 (6), 999-1015.

Jiménez, M. C. (2014). Los tres lenguajes matemáticos más utilizados en economía. Economíalnforma, 2014(388), 36-49.

Jiménez, M. M., García, V. J. ., \& Molina, L. M. (2011). Validation of an instrument to measure absorptive capacity.Technovation, 31(5-6), 190202. doi:10.1016/j.technovation.2010.12.002

Jiménez-Barrionuevo, M., García-Morales, V., and Molina, L. (2011). Validation of an instrument to measure absorptive capacity.Technovation 31, 190-202.

Johnson G. \&Scholes K. (2001). Dirección estratégica. (5a Ed.). Madrid: Prentice Hall.

Johnson, G. \&Scholes, K. (1997). Dirección estratégica: análisis de la estrategia de las organizaciones (3a Ed.). Madrid: Prentice Hall.

Johnson, G., Scholes, K. \& Whittington, R. (2006).Dirección estratégica. Madrid: Pearson Education.

Jones, R. E. ., Wyoming Univ.; Casper, W. Y. ., \& Jacobs, L. W. (1991).Consequences of centralized strategic decision making in high technology firms.
Technology Management: the New International Language (pp. 27-31). IEEE CONFERENCE PUBLICATIONS.

Lane, P.; Koka, B. and Pathak, S. (2006). The reification of absorptive capacity: A critical review and rejuvenation of the construct. Academy of Management Review, 31 (4), 833-863.

Liao, S., Fei, W., and Chen, C. (2007). Knowledge sharing, absorptive capacity, and innovation capability: An empirical study of Taiwan's knowledgeintensive industries. Journal of Information

Manso C., F.J. (1991). Curso de dirección estratégica comercial. Madrid: ESIC Editorial.

Marquez, A. C., \&Blanchar, C. (2006). A Decision Support System for evaluating operations investments in high-technology business. Decision Support Systems, 41(2), 472-487. doi:10.1016/j.dss.2004.08.012

Máynez-Guaderrama, A., Cabazos-Arroyo, J., and NuñoDe La Parra, J. (2012). La influencia de la cultura organizacional and la absorptivecapacity sobre la transferencia de knowledge tácito intraorganizacional. [The influence of organizational culture and the absorptive capacity of the tacit intra-organizational knowledge transfer.] EstudiosGerenciales, 28 (Special Edition) 191211.

Medina, V. H., \&Verástegui, F. A. (2015). La gestión de conocimiento y su aplicabilidad en las organizaciones. Ingenierías\& Amazonia,6(1).

Minbaeva, D.; Pedersen, T.; Bjorkman, I.; Fey, C., and Park, H. (2003).MNC knowledge transfer, subsidiary absorptive capacity, and HRM. Journal of International Business Studies, 34 (6), 586-599.

Mintzberg, H. (1978). Patterns in strategy formation.En: Managemente Science, XXIV(9): 934-948.

Mintzberg, H. (1987). The Strategy Concept I: Five Ps For Strategy. En: Fall 1987 California Management Review.

Mintzberg, H., (1990). "The Design School: Reconsidering the Basic Premises of Strategic Management". Strategic Management Journal 11 (3), 171-195.

Mintzberg, H., Quinn, J.B. \&Ghoshal, S. (1999). El proceso estratégico. (Edición europea revisada). España: Prentice Hall.

Montoya Restrepo, I. A. (2010) Una contribución a la comprensión de las estrategias deliberadas y emergentes de las organizaciones, desde una perspectiva evolutiva (Doctoral dissertation, Universidad Nacional de Colombia). Disponible en:

http://www.bdigital.unal.edu.co/2141/1/UNA_C 
ONTRIBUCI\%C3\%93N_A LA COMPRENSI\%C3\% 93N_DE LAS_ESTRATEGIAS_DELIBER.pdf

Montoya, I. \& Montoya, L. (2003). El direccionamiento estratégico y su aplicación en los sistemas complejos y en la gerencia ambiental. En: Innovar, 13(21): 81-104.

Montoya, I. (2009). La formación de la estrategia en Mintzberg y las posibilidades de su aportación para el futuro. RevistaFacultadCienciasEconómicas Universidad Militar Nueva Granada, XVII(2), 2344.

Montoya, I. (2011). El Campo de la Dirección Estratégica. Medellín: Universidad Nacional de Colombia Sede Medellín.

Montoya, I., \& Montoya, A. (2003). El direccionamiento estratégico y su aplicación en los sistemas complejos y en la gerencia. Innovar. Revista de Ciencias, 021, 81-104. Retrievedfrom http://redalyc.uaemex.mx/redalyc/pdf/818/818 02107.pdf

Mowery, D., and Oxley, J. (1995). Inward technologies transfer and competitiveness: The role of national innovation systems, Cambridge Journal of Economics, 19 (1), 67-93.

Murovec, N. and Prodan, I. (2009). Absorptive capacity, its determinants, and influence on innovation output: cross-cultural validation of the structural model, Technovation, 29 (12), 859-872.

Nugraha, T. (2011). Searching for Potential and Realized Absorptive Capacity of the Firm: The Case of Japanese Joint Ventures in Indonesia. The IUP Journal of Knowledge Management, Vol. IX (4), 54-75.

Olalla, M. F., Sandulli, F. D., Menéndez, J. F., \& Duarte, A. R. (2014). ¿ Cómo afectan las características de la industria a la relación entre la capacidad de I+ D y la adopción de innovación abierta de entrada?.Economía industrial, (391), 23-32.

Oliver, J. L. H., \& Ripoll, F. S. (2014). I+ D y capacidad de absorción: evidencia empírica en España. Economía industrial, (391), 43-48.

Pandza, K., \& Holt, R. (2007).Absorptive and transformative capacities in nanotechnology innovation systems. Journal of Engineering and Technology Management, 24(4), 347-365. doi:10.1016/j.jengtecman.2007.09.007

Patterson, W., and Ambrosini, V. (2015).Configuring absorptive capacity as a key process for research intensive firms. 41 Technovation, 36/37, 77-89.

Ritala, P., and Hurmelinna, P. (2013).Incremental and Radical Innovation in Coopetition - The Role of
Absorptive Capacity and Appropriability. Journal of Product Innovation Management, 30(1), 154-169.

Ronda, P.G. \&Marcané, J.A. (2004). Dirección estratégica integrada. Un enfoque para integrar los niveles estratégico, táctico y operativo. Instituto de InformaciónCientífica y Tecnológica, (19), 4.

Rothaermel, F. T., \&Thursby, M. (2007). The nanotech versus the biotech revolution: Sources of productivity in incumbent firm research. Research Policy, 36(6), 832-849. doi:10.1016/j.respol.2007.02.008

Saloner, G., Shepard, A. \&Podolny, J. (2005).Administración estratégica. México: LimusaWiley.

Schildt, H., Keil, T., and Maula, M (2012).The temporal effects of relative and firm-level absorptive capacity on interorganizational learning. Strategic Management Journal, 33, 1154-1173.

Seo, Y., Chae, S., and Lee, K. (2015). The impact of absorptive capacity, exploration, and exploitation on individual creativity: Moderating effect of subjective well-being. Computers in Human Behavior, 42, 68-82.

Spithoven, A., Clarysse, B., \&Knockaert, M. (2010).Building absorptive capacity to organise inbound open innovation in traditional industries.Technovation, 30(2), 130-141. doi:10.1016/j.technovation.2009.08.004

Szulanski, G. (1996). Exploring internal stickiness: Impediments to the transfer of best practice within the firm. Strategic Management Journal, 17, 27-43.

Taylor, F. W., \&Fayol, H. (1981).Principios de la administración científica. El Ateneo.

Tether, B. S. (2002). Who co-operates for innovation, and why An empirical analysis. Resarch Policy, 31(6), 947-967.

Thompson, A. \& Strickland, A. J. (1998).Dirección y administración estratégicas. México: McGraw Hill.

Todorova, G., and Durisin, G. (2007). Absorptive capacity: Valuing a reconceptualization. Academy of Management Review, 32 (3), 774-786.

Vega-Jurado, J., Gutiérrez-Gracia, A., and Fernández-deLucio, I. (2008) Analyzing the determinants of firm's absorptive capacity: beyond R\&D. R\&D Management, 38 (4), 392-405. 
Villanueva, A. Montoya, I. Montoya, L.. (2015). Análisis De Las Decisiones Estratégicas: Caso Une Epm Telecomunicaciones. RevistaFacultad de CienciasEconómicas: Investigación y Reflexión, 23(1), 267-287.

Von Glinow, M., Young, A., \&Mohrman., S. A. (1990). Managing complexity in high technology organizations. New York: Oxford Press.

Waranantakul, W., and Ussahawanitchakit, M. (2012). Marketing knowledge absorptive capacity and marketing performance: an empirical investigation of food product exporting businesses in Thailand. International Journal of Business Strategy, 12 (3), 62-80.

Wheelen, T.L. \& Hunger, J.D. (2007).Administración estratégica y política de negocios: conceptos y casos. (10a ed.). México: Pearson Education.

Xiong, G., and Bharadwaj, S. (2011). Social Capital of Young Technology Firms and Their IPO Values: The Complementary Role of Relevant Absorptive Capacity. Journal of Marketing, 75 (6), 87-104.

Zahra, S., and George, G. (2002). Absorptive capacity: A review, reconceptualization, and extension, Academy of Management Review, 27 (2), 185203. 